\title{
« Entrar con el otro y salir con sigo » : nouvelles recherches sur les missions d'évangélisation modernes
}

\section{Pierre Antoine Fabre}

\section{(2) OpenEdition Journals \\ Édition électronique \\ URL : http://journals.openedition.org/assr/26292 \\ DOI : $10.4000 /$ assr.26292 \\ ISSN : $1777-5825$ \\ Éditeur \\ Éditions de l'EHESS}

\section{Édition imprimée}

Date de publication : 31 décembre 2014

Pagination : 43-54

ISBN : 978-2-7132-2467-6

ISSN : 0335-5985

Référence électronique

Pierre Antoine Fabre, « «Entrar con el otro y salir con sigo » : nouvelles recherches sur les missions d'évangélisation modernes ", Archives de sciences sociales des religions [En ligne], 168 | 2014, mis en ligne le 30 mars 2018, consulté le 03 mai 2019. URL : http://journals.openedition.org/assr/26292 ; DOI : 10.4000/assr.26292

Ce document a été généré automatiquement le 3 mai 2019.

(C) Archives de sciences sociales des religions 


\title{
« Entrar con el otro y salir con sigo " : nouvelles recherches sur les missions d'évangélisation modernes
}

\author{
Pierre Antoine Fabre
}

\section{RÉFÉRENCE}

Ragon Pierre (éd.), Nouveaux chrétiens, nouvelles chrétientés, Paris, Presses de l'Université de Paris-Ouest, coll. «Bibliothèque historique », 2014, 382 p., et de plusieurs autres ouvrages et travaux récents.

\section{NOTE DE L'AUTEUR}

« Entrer dans l'autre et sortir de soi-même », Ignace de Loyola, Instruction pour une mission en Irlande, septembre 1541. Ines G. Zupanov et moi-même préparons une étude sur les nombreux développements et variations de cette formule, fondatrice pour le discours de la Compagnie de Jésus.

1 Un spectre hante les pages de ce livre ${ }^{2}$ : le spectre du syncrétisme. Chassez-le par la porte, il revient par la fenêtre! Cette insistance est étrange, alors que, depuis des années déjà, le concept a été sévèrement étrillé, soit parce qu'il dissimulait des contradictions et des confrontations symboliques et rituelles que la notion de syncrétisme tendait à recouvrir d'un voile parfois presque irénique ${ }^{3}$, soit parce qu'au contraire, il maintenait ou plus encore présupposait des entités culturelles substantielles, séparables et identifiables, dont la combinaison sans confusion serait le syncrétisme. ${ }^{4}$ Les contributions de l'ouvrage suggèrent cependant une explication à la persistance d'une notion aussi percluse : d'une manière parfois presque imperceptible, parce que le temps n'y est pas le même, le calendrier moins serré, les repères politiques, militaires, diplomatiques, culturels moins denses, nous nous sommes déplacés sur presque quatre siècles, dans une modernité de 
très longue durée, depuis la «malle magique » ouverte par Eric Roulet, découverte en 1541 dans un village du Mexique central, jusqu'aux confréries de l'archevêché de Mexico, étudiées par Nadine Béligand jusqu'aux dernières années du $\mathrm{xVIII}^{\mathrm{e}}$ siècle. Or ce qui pouvait relever d'un syncrétisme prend souvent consistance entre deux moments, dans deux phases différentes des rapports de force entre des cultures exogènes et endogènes, multiples chacune et qui, vues de surplomb présentaient le visage d'un syncrétisme, dont chacune des configurations particulières pouvait elle-même offrir un trait. Aussi peut-on se demander si le syncrétisme ne serait pas l'avatar dans les études américaines d'un débat lui aussi insubmersible entre une approche historienne et une approche structurale, le modèle du syncrétisme sauvant, sous l'angle structural d'un bricolage, ce que l'approche historienne déconstruirait en fonction des situations et, plus spécifiquement, des savoirs situés en fonction desquels le discours de l'historien se constitue. Nous retrouverons dans ce qui suit de nombreux exemples de ce conflit, que l'extinction progressive des grands ébranlements de la connaissance historique n'a pas suffi à réduire, mais qui prend aujourd'hui le détour paradoxalement suranné de ce mot trop commode ou au contraire définitivement inconfortable de «syncrétisme ».

2 Au-delà de cette remarque générale, je distribuerai ce qui suit selon trois ensembles : ce que ces travaux font apparaître comme des acquis décisifs de la recherche ; les problèmes qui me semblent subsister ; les propositions que ces difficultés inspirent.

\section{Acquis}

3 1. L'histoire des missions d'évangélisation est aujourd'hui inscrite dans celle des sociétés américaines telles qu'elles se constituent à égale distance de l'Europe et de l'Amérique précolombienne. L'usage fréquent de la notion de «frontière » tient dans ce volume à ce que la mission de frontière - qui s'exerce aux confins des empires coloniaux et contribue à définir ces confins en même temps que la mission opère là où chacun des pouvoirs centraux trouve sa limite - est aussi une nouvelle frontière de l'historiographie des missions. Frontière sans cesse repoussée, au fur et à mesure que l'on découvre comment les expéditions ou implantations les plus apparemment anarchiques (autonomes, autogouvernées, autodisciplinées, etc.) furent souvent étroitement négociées entre les organisations religieuses présentes, les autorités civiles, les agents économiques. ${ }^{5}$ Cette inscription sociale fait du missionnaire, envoyé à ce titre par son ordre, l'un seulement des acteurs religieux sur la scène coloniale, entre le clergé séculier et les dévots laïcs; elle fait également de l'action proprement missionnaire de ce missionnaire, l'évangélisation des non-chrétiens, une dimension seulement de son activité. Le cas des Augustins récollets de Nouvelle France, étudié par Caroline Galland, est instructif à cet égard, puisque le projet de peuplement s'y donne comme une condition du projet de civilisation, lui-même prémice d'un processus d'évangélisation.

Il faudrait également mobiliser ici toute une série de travaux récents qui ont tenté d'éclairer par quelles voies les ordres religieux européens, et singulièrement des ordres contemplatifs, ont pu effectuer une conversion missionnaire et parvenir cependant à ne pas déroger à leurs principes fondamentaux, en concevant l'avancée missionnaire comme un stade suprême du retrait du monde. C'est par exemple le cas des tentatives des carmes déchaux Jerónimo Gracían et Tomás de Gesú, sur lesquels des travaux manquent encore, qui seraient fondamentaux pour inscrire l'histoire des missions d'évangélisation dans une histoire spirituelle d'échelle mondiale. Giovanni Pizzorusso, l'auteur des travaux les plus 
importants sur la Propaganda Fide, a montré le poids de ces discours dans la genèse de cette institution romaine (voir l'entrée "Francesco Ingoli», premier préfet de la Propaganda en 1622, in Dizionario biografico degli italiani). Il faut également situer dans cette même perspective les conflits entre apostolat et enseignement, qui ne concernent pas seulement les communautés religieuses européennes ou le rapport entre ces communautés et le "monde missionnaire ", mais bien ce monde comme tel, dans lequel la formation des élites coloniales et créoles devient rapidement un terrain central (voir les travaux d'Antonella Romano, en particulier entre d'autres «Les premiers enseignements scientifiques en Nouvelle Espagne", in L.M.Carolino et C. Ziller Camenietski éds., Jesuitas, ensino e ciencia, p. 181-201).

5 2. La catégorie des dévots laïcs fait venir au premier plan le phénomène des confréries ou congrégations laïques ${ }^{6}$. Notre volume contribue certainement à montrer son importance, à travers les contributions de Nadine Beligand, ou de Pierre Ragon. Je voudrais insister ici sur un aspect, celui des confréries comme instruments de mesure des respirations et des intermittences des choix d'intercession. Le système dévotionnel catholique organise une constellation de relais pour l'accès à Dieu, puissance en acte. Le choix n'est pas d'user ou non de ces relais (on sait que le fil tranchant de l'orthodoxie passe sur cette ligne), mais il est entre ces relais: le dévot, de ce point de vue, distribue ses faveurs comme Dieu distribuera ses grâces. Tout le problème est de savoir selon quels mobiles l'astre des médiateurs s'élève ou pâlit, pourquoi il s'épuise, quel est le rythme de ces scansions. Nadine Beligand propose plusieurs hypothèses convaincantes : d'une part sur la scansion d'un privilège christo-marial et des cultes des saints, comme dans une sorte d'alternance déflationniste et inflationniste sur le marché de l'intercession, dynamique réfléchie au sein même de chacun des cultes avec une démultiplication interne du culte de la Passion, par exemple, dans chacune de ses stations, etc.; d'autre part sur la compénétration progressive des cultes ruraux et urbains, n'excluant pas une hiérarchisation interne à ces partages, dans l'organisation des confréries, par exemple. ${ }^{7}$

6 3. Un troisième champ, le plus difficile sans doute, s'impose enfin dans ces pages : celui, à double face, de la langue et de l'écriture. La question de la langue est réellement venue au jour depuis une dizaine d'années, pour une raison historiographique majeure : à partir du moment où l'on s'interrogeait sur la figure du missionnaire comme telle, et non pas seulement sur les traces qu'il avait laissées (ce qu'on appelle souvent une préhistoire de l'ethnographie) ou sur les institutions dont ils étaient les messagers, il devenait impérieux de savoir comment ces «médiateurs» s'étaient placés dans l'intervalle immense qui séparait les langues indigènes des langues européennes. C'est ainsi que nous sommes passés - dans les travaux de Charlotte de Castelnau, ou dans ceux de Juan Carlos Estenssoro $^{8}$ ou, tout aussi bien dans les recherches de Bernadette Majorana sur l'Italie centrale, les "Indes d'ici », où l'on parlait une langue tout aussi opaque que le quechua ou le mapuche pour un jeune jésuite sorti des collèges de Milan ou de Modène - de l'évidence à peine soupçonnée d'une sorte de pentecôtisme néo-apostolique à la découverte des apprentissages, laborieux, incertains, des langues locales, ou d'une partie d'entre elles érigées en "langues générales »; le problème s'est alors déplacé : l'intention de parler les langues n'est plus en doute, d'innombrables documents en témoignent, mais l'efficacité de cette parole reste souvent une énigme - partiellement résolue par les informateurs, dont le rôle apparait aujourd'hui aussi central qu'il le sera, quelques siècles plus tard, dans les missions ethnographiques. Deux contributions rendent compte, par deux voies très différentes, de cette difficulté. Celle de Moises Franco Mendoza, qui analyse le 
discours rhétorique comme cadre d'intelligibilité de la langue (et non pas le contraire), et celle de Frédéric Duchesne qui montre comment certaines notions de la langue quechua fonctionnent comme des «notions générales ", pourrait-on dire, aptes ou pensées aptes à ressaisir un particulier divers, et sont déjà en elles-mêmes une première forme de traduction de ce divers (comme la notion de «huaca» pourrait en donner l'exemple, premier palier vers la notion d'«idole», elle-même condition de possibilité de l'« extirpation de l'idolâtrie »).

7 Bérénice Gaillemin porte dans ce livre, avec l'étude des manuscrits réalisés sous la conduite du franciscain Jacopo di Testera, dits testériens, un débat important, sur lequel un ouvrage récent de Silvia Sebastiani a également mis l'accent ${ }^{9}$, concernant la définition des pictogrammes préhispaniques, alphabet ou idéogramme. ${ }^{10}$ L'enseignement le plus remarquable de ces manuscrits est sans doute l'insistance des chrétiens d'Amérique à replacer leur pas dans celui des écritures qui les avaient précédés, pour les modeler à leur «image », sans doute. Mais, dans le détail de leur geste, c'est ce geste qui se trouve modelé et qui brouille les frontières entre une graphie essentiellement précolombienne et une graphie essentiellement coloniale.

De tout cela ressort, presque trop massivement aujourd'hui pour être toujours perçue, une forte autonomie des espaces américains, dont témoigne le recadrage de la notion de mission au sein même de ces espaces comme un autre découpage intérieur. ${ }^{11}$ Non pas que les modèles européens ne soient pas directeurs dans la construction des cultures chrétiennes américaines (discours/écritures, lieux, figures), mais ces modèles doivent devenir américains pour continuer d'opérer: le cas de la Vierge de Loreto étudié par Luisa Elena Alcala est significatif de cette migration. Il en est même un paradigme puisque cette Vierge représente la Santa Casa dont elle est issue (et qui est du reste elle-même le développement d'une statue primitive) et que, comme nous le savons, cette Casa a d'abord été transportée depuis l'Orient jusqu'à l'Europe ${ }^{12}$ : le voyage de la Vierge en Nouvelle-Espagne n'est que la poursuite d'un vaste mouvement de l'Orient vers l'Occident dont la station européenne n'aura été qu'une étape.

Inversement, l'Europe commence de s'affirmer, dans l'étape où nous sommes, comme une «aire culturelle » entre d'autres, ni plus, ni moins, c'est-à-dire non pas comme une métropole soustraite à ses propres découpages ${ }^{13}$ et non plus comme une aire culturelle comme une autre, dont on oublierait l'expansionnisme, la domination, l'universalisme spécifique, catholique ou autrement idéologique. Mais les effets de miroir sont instructifs ${ }^{14}$ pour progressivement dessiner une cartographie des interactions, dans lesquelles l'Europe n'est plus seulement motrice mais aussi réceptrice: autant d'évolutions qui deviennent familières, en particulier à travers l'histoire de la Compagnie de Jésus et les répercussions européennes de son histoire mondiale ${ }^{15}$, mais qui marquent, non sans de nombreuses variations, l'intégration et la reprise critique récentes des Subaltern Studies.

\section{Problèmes}

\section{Le « monde»}

10 Tout ce qui précède nous propulse évidemment sur une scène mondiale: un grand nombre des objets ou des notions évoqués dans les pages de ce livre mérite, en effet, d'être considéré non pas comme spécifiquement attaché aux espaces américains, mais comme une donnée générale dont les spécificités américaines sont à construire ; et ceci 
non seulement par rapport à l'Europe mais par rapport à d'autres espaces extraeuropéens. Le grand dossier de la "querelle des rites", abordé par plusieurs contributions à travers le baptême des Indiens ${ }^{16}$, relève de cette mondialité : comment cette querelle touche-t-elle l'ensemble des lieux de confrontation et d'articulation de pratiques d'initiation et de reconnaissance concurrentes, c'est-à-dire aussi bien les grandes cités chinoises du xviie siècle que les tribus amazoniennes de la même époque ; et pourquoi l'une seulement de ces terres a-t-elle bénéficié de la promotion apostolique, théologique, philosophique de la « querelle des rites chinoise »? Autrement dit, comment mondialiser l'horizon de l'enquête sans constituer des continuités fallacieuses ou des équivalences trompeuses? Comment historiciser en permanence l'espace qui s'offre à nous dans une disponibilité croissante avec la prolifération des recherches multiplement localisées? Le problème est central, aussi bien pour ce que l'on peut constater de la limite des exercices de " coupe " planétaire, comme la vaste et finalement incertaine entreprise récente de L'histoire du monde au xve siècle ${ }^{17}$, que pour l'ouverture des possibles, dont le présent volume rend bien compte : on peut rêver d'une histoire mondiale des confréries chrétiennes, dans l'orientation de l'enquête de Nadine Béligand, ou d'une histoire mondiale de la fonction «pacificatrice » des clercs, dont Bernard Grumberg donne ici un exemple pour la Nouvelle-Espagne, et dont Anne Bonzon, dans des recherches en cours ${ }^{18}$, montre l'importance dans la France de la Réforme catholique: ou encore de la "superstition » et de l'« idolâtrie », notions tout aussi communes en Amérique (comme le montre Frédéric Duchesne) qu'en Europe à la même époque ; et inversement, des limites du désenchantement des images, dont témoignent aussi bien la gestation difficile du Décret du Concile de Trente sur les conditions d'un culte légitime des saintes images ${ }^{19}$ que la passionnante enquête de Christophe Giudicelli et Pierre Ragon, très utile complément à ce volume, sur «Les martyrs ou la vierge? Frères martyrs et images outragées dans le Mexique du Nord (xvi-xviie siècles) $»^{20}$, qui montre comment la promotion des images au martyre, qui les incarne et les charge d'une sacralité propre, bien loin des recommandations du Concile, est le recours de l'impossible « martyre sans bourreau » des premiers missionnaires; de la même manière que dans sa contribution à ce volume, Ragon comprend la promotion des causes de sainteté dans l'église liménienne (dès 1594) comme une explication possible de la moindre fréquence des images miraculeuses, en comparaison du Mexique. ${ }^{21}$ Mais l'essentiel, me semble-t-il, il faut le répéter ici, est de construire une histoire faite de mains d'homme, qui ne mime pas le vol de l'oiseau et qui, au contraire, repère ce qui, dans le monde tel qu'il se constitue, fabrique de la mondialité par l'articulation des lieux et des problèmes.

\section{La « culture chrétienne »}

11 Elle ne peut pas être convoquée sans réfléchir sur le mode de totalisation qu'elle représente concernant des institutions et des hommes qui, eux-mêmes, se situent dans une tradition immense dans laquelle les époques et les générations se confondent souvent, comme peuvent se confondre l'histoire de l'Église comme formation historique et les récits qui la fondent. Ce livre nous donne de nombreux exemples de ce fleuve. Il faut, là aussi, savoir situer les eaux du fleuve, qui ne repassent jamais sous le même pont: comment, à un certain moment, le renoncement, par exemple, à la représentation sacrificielle du Fils de Dieu crucifié a pu être théologiquement pensable pour des chrétiens alors qu'elle ne le serait sans doute plus aujourd'hui ? Comment la continuité vétéro- et néo-testamentaire (dont témoignent par exemple la combinaison des séries de 
péchés étudiées par Moises Franco Mendoza) a pu s'inscrire dans un contexte qui était aussi celui de la réduction à la révélation chrétienne des Juifs de Chine, de la conversion des tribus perdues d'Israël (auquel renvoie Liliane Crété dans le cadre de son enquête - ce n'est pas un hasard - sur le pasteur Eliot), et, à l'échelle de la construction théorique, des diverses formes de figurisme qui marquent la théologie chrétienne des $\mathrm{XVII}^{\mathrm{e}}$ et $\mathrm{XVIII}^{\mathrm{e}}$ siècles.

\section{Les schismes américains}

12 Sous ce troisième titre deux problèmes se tiennent, qui me semblent l'un comme l'autre aussi lancinants que fugitivement exposés au fil de ces pages ; et j'ai souvent songé, à leur lecture, aux initiatives qu'il faudrait prendre pour les déployer pleinement. Le premier concerne le destin américain des schismes $\mathrm{du} \mathrm{XVI}{ }^{\mathrm{e}}$ siècle européen. Pour la première fois peut-être, tout au moins pour la période moderne, un même ensemble accueille des études portant sur les zones d'influence catholique et protestante: Lucia Bergamasco, Liliane Crété, Germaine Lemétayer ${ }^{22}$ ont ouvert un horizon souvent absent des recherches françaises jusqu'ici. Mais reste à venir une interrogation sur les rapports de ces zones d'influence. Ces schismes sont-ils exportés à l'identique? Comment les frontières bougent-elles, si elles bougent, dans des espaces différents? Pierre Ragon a récemment montré23 par une analyse comparée de la Nouvelle-Espagne et de l'Amérique du Nord comment les postulats théologiques de la Réforme conduisaient à des stratégies de conquête dans lesquelles la part de la conversion comme bras religieux de la civilisation ne pouvait pas être la même dans son principe. Mais, de la même manière que la possibilité de la conversion comme processus d'adhésion progressive d'abord manifeste par des signes extérieurs n'a pas exclu la contrainte, religieuse et plus généralement civile, l'obstacle théologique a-t-il exclu toute forme de communication religieuse ? Et, par ailleurs, le domaine de la "civilité » n'a-t-il pas ouvert des «domaines partagés » entre les confessions? La contribution de Germaine Lemétayer va dans ce sens en montrant comme le pasteur Pierre Moreau, au Brésil dans les années 1650, attache au travail une importance que l'on retrouve, avec des implications différentes (dans le second cas la justification de la servitude), dans les entreprises de la Compagnie de Jésus à la même époque et dans la même région d'Amérique. Plus généralement, la part de la civilité dans la civilisation des Indiens d'Amérique - ou des «Indiens» d'Asie ou des Chinois - révèle autant de clivages intra (comme le feront bien voir les «querelles des rites ») qu'interconfessionnels. Les recherches de Lucia Bergamasco sur la part inverse de ce qu'elle appelle le «merveilleux» - terme éloquemment médiéval - dans les protestantismes américains ouvrent d'autres perspectives de croisement, largement inexplorées.

Aux schismes d'importation, il est nécessaire, comme cela ressort clairement de la contribution de Philippe Delisle sur Haïti, d'ajouter des schismes chrétiens spécifiquement américains, dont la solution du syncrétisme ne suffit pas à rendre raison. La longue durée dans laquelle se place ce volume permet justement une autre hypothèse : celle d'une évolution du christianisme, dans sa doctrine, dans ses pratiques, dans son inscription historique, qui en Amérique, comme en Europe, produirait des formes nouvelles, dont nous pourrions chercher jusqu'au $\mathrm{xx}^{\mathrm{e}}$ siècle les développements, dans ce qu'on a appelé la théologie de la libération, par exemple, qui ne se confond nullement tout au contraire même - avec un métissage, et qui, cependant, peut bien recevoir le nom 
de schisme, si l'on considère le jugement que porta sur elle le magistère romain sous le pontificat de Jean-Paul II.

\section{Propositions}

14 Comment aller de l'avant? On pourrait suggérer pour finir quatre orientations de méthode, toutes inspirées par les contributions de ce volume.

\section{Cultiver une inquiétude des sources}

15 Toute source en elle-même est fascinante dans la réalité que, par éclairs, elle fait apercevoir, et se replie cependant inexorablement sur elle-même. La source montre la lune, l'idiot regarde la source : il faut être idiot, comme la plupart de ces contributions le sont en découvrant comment les procès, les mémoires, les récits, les lettres produisent la réalité dont ils rendent compte. La seule solution est dans une intertextualité qui, elle, peut faire apercevoir au défaut de chacune de ces sources ce qui les éclaire par d'autres, comme quand Delphine Tempère découvre l'importance extrême de la vie maritime comme entre-deux-mondes dans la mesure même où celle-ci n'apparaît qu'à la marge de récits dont la finalité est de dénier cet entre-deux-mondes, comme intervalle, comme utopie et comme une société dans laquelle le seul maître après Dieu n'est pas son représentant, mais le capitaine. Dans cette intertextualité, les sources visuelles doivent éveiller davantage encore, en proportion de leur aveuglante évidence, les soupçons de l'idiot, en particulier pour des cultures dans lesquelles cette évidence de l'image- comme «bible des ignorants »- n'en est pas une ${ }^{24}$.

Les possibilités offertes aujourd'hui par la publication numérique devraient permettre, dans les années à venir, d'augmenter le profit de cette inquiétude épistémologique (très vive par exemple dans la remarquable contribution de Soizig Groguennec, au sujet des sources inquisitoriales) et en particulier de ne pas choisir entre deux grandes voies pour l'intelligence de sources qui toutes, d'une manière ou d'une autre, s'inscrivent dans des genres (littéraires, institutionnels) : mettre en série les «individus » répondant à ces genres pour en faire apparaître les constances rhétoriques, ou construire la confrontation entre ces genres pour déduire les réalités que chacun de ces genres transforme.

\section{Explorer les transversalités mondiales contemporaines}

17 Si l'on veut éviter de projeter un "monde ", tel qu'il est devenu le nôtre (dont l'unité reste à prouver), sur la pluralité des mondes anciens (le point de vue de la Chine du XVII ${ }^{\mathrm{e}}$ siècle sur le monde, le point de vue des grands empires de l'Afrique précoloniale sur le monde, etc.), et cependant concevoir ce qui, dans cette pluralité, articule ces mondes dans un monde unique, la meilleure voie, et la plus difficile évidemment, semble être ${ }^{25} \mathrm{de}$ repérer un horizon de monde à hauteur d'homme, comme c'est le cas dans la contribution de Bérénice Gaillemain, où l'ordonnancement des péchés participe d'une histoire comparée, produite par les "acteurs » eux-mêmes ${ }^{26}$. On pourrait citer le cas aussi, dans l'océan des bribes qu'il faut ici sauver, de la Difesa dei missionnari della Compagnia di Gesu de Giovanni Battista de Benedictis, 1700 : «Que vienne en Europe un indien idolâtre et qu'il voit le culte que, dans les églises catholiques, on porte aux images sacrées, qu'il voit les fidèles jetés à genoux devant elles, les baiser, les adorer, les supplier, il jugera facilement 
qu'elles sont nos idoles... ». Benedictis convoque, d'une manière quelque peu inattendue dans ce pamphlet, à l'acmé de la querelle des rites de Chine, un «idolâtre » des Indes occidentales - témoignant fortement ici de l'horizon mondial d'un polémiste ordinaire de l'Italie de la fin du XVII ${ }^{\mathrm{e}}$ siècle - pour laver l'« Indien » de Chine du soupçon d'idolâtrie. ${ }^{27}$

\section{Déjouer les évidences anthropologiques}

Les contributions de ce volume sont frappantes par la récurrence d'un thème, ou plus que d'un thème, d'un abîme : la mort. ${ }^{28}$ La mort dont on ne sait rien. Or tout aussi frappante est l'extraordinaire diversité des voies par lesquelles ce non-savoir est compensé. De quoi parle-t-on quand on parle de la mort? Des assurances sur la mort des confréries étudiées par Nadine Beligand ? Du traitement posthume des corps à Arequipa au XVIII ${ }^{\mathrm{e}}$ siècle ? Des usages funéraires andins, qui ne sont réductibles à des rituels (rapportés à ces usages), ni à des cultes (rapportés à leur objet) ? Il faut passer par une déconstruction de cet abîme pour pouvoir reconstruire terme à terme les conditions de possibilité d'une anthropologie comparée des humanités mortelles.

\section{Recharger de savoir les concepts théologiques}

ne contribution de ce volume, celle de Maristela de Andrade, accompagnée lors du colloque de 2011 d'une intervention d'Aliocha Maldavsky, a fait apparaître, non sans écho indirect aux travaux de Michel Foucault sur les ateliers de charité européens, la complexité de la vertu théologale de charité, qui recouvre une grande variété de pratiques dont elle produit en quelque sorte la théorie générale, qu'il s'agisse de la charité libérale de la donation comme rachat de soi (étudiée par A. Maldavsky avec les legs des encomenderos du Pérou colonial), ou de la charité morale et sociale dans la mise au travail d'une communauté rachetée par sa vertu, comme dans les « maisons de charité " brésiliennes du XIX ${ }^{e}$ siècle évoquées par M. de Andrade. ${ }^{29}$ La vertu théologale de charité, que l'on retrouve par ailleurs dans le Dialogo de doctrina cristiana de Gilberti relu par Moises Franco, intervient ici à un autre niveau, non pas comme objet mais comme catégorie efficace pour concevoir l'articulation de l'individualité et de la communauté dans l'Amérique coloniale.

Sur ce dernier point, l'évolution des recherches sur l'histoire de l'évangélisation américaine rejoint un trait plus général des études sur les faits religieux: le désenclavement de la théologie, qui n'est plus écartée en tant qu'elle s'octroierait une position de domination sur les autres sciences dans le cadre professionnel et confessionnel d'une institution elle-même prétendant à la domination, mais qui est accueillie comme l'un de ces savoirs situés que j'évoquais en commençant, théorie d'une pratique, et théorie pouvant elle-même faire l'objet d'une histoire à travers celle de ses producteurs, de ses relais, de l'autonomie intellectuelle de ses héritages, etc. La missiologie, qui a longtemps été le cheval de Troie de la théologie dans l'interprétation des aventures extra-européennes de l'église chrétienne, laisserait maintenant la place à l'histoire d'une théologie en mission, dont nous avons encore beaucoup à découvrir. Les recherches en cours de Jean-Pascal Gay (Université de Strasbourg) pour son habilitation de recherches seront ici d'un grand apport: il montre le rôle des théologiens comme relais de l'autorité ecclésiastique dans les marges de l'Église, et l'importance de ce rôle alors même que ces théologiens sont souvent soustraits à des carrières académiques 
européennes par les exigences du recrutement missionnaire et qu'ils sont donc tout aussi méconnus dans leur milieu d'origine que reconnus dans leurs terres d'adoption - le tout permettant évidemment de comprendre le relatif oubli dans lequel le temps a plongé ces spécialistes inaboutis, mais cependant accomplis. ${ }^{30}$

\section{NOTES}

2. Fruit d'un colloque réuni à Paris-Ouest en 2011, l'ouvrage très soigneusement édité par Pierre Ragon présente un assez large panorama des recherches actuelles dans le domaine de l'histoire de l'évangélisation, tout au moins dans le monde francophone. Il nous a donc semblé mériter une note critique, que l'on enrichira au fil de la lecture par des références complémentaires.

3. C'est, me semble-t-il, cette première critique que vise Pierre Ragon lorsque, dans ses recherches sur les cultes reliquaires chrétiens en Nouvelle-Espagne, il montre comment ces cultes s'imposent, et doivent sans doute s'imposer pour ne pas s'exposer à des réappropriations incontrôlables : autrement dit, pour contrer le risque d'un synchrétisme, qui serait ainsi du côté d'une histoire des savoirs pratiques que d'un laboratoire des catégories historiographiques.

4. La notion de métissage, telle que Serge Gruzinski en a déployé les fastes, avait pour projet, largement accompli, de brouiller ces contours implicites. Mais il n'a pas résolu tout-à-fait l'autre difficulté du syncrétisme.

5. Voir par exemple sur ce point les recherches récentes de Camila Loureiro Dias sur le Maranao (dans un doctorat en cours de publication). Voir également le grand travail de Joé del Rey Fajardo sur Los jesuitas en Venezuela. Caracas/Bogotá/San Cristóbal, Universidad Católica Andrés Bello/ Pontificia Universidad Javeriana/Academia Nacional de la Historia/Fundación Fondo Editorial Simón Rodríguez, 2006-2011 (6 vols.). Il faut enfin rappeler les travaux déjà classiques de Dauril Alden, The Making of an Enterprise. The Society of Jesus in Portugal, its Empire and Beyond, Stanford, Stanford University Press, 1996, et Carlos Zeron, Lignes de foi. La compagnie de Jésus et l'esclavage dans le processus de formation de la société coloniale en Amérique portugaise (XVI $-\mathrm{XVII}{ }^{e}$ siècles), Paris, Champion, 2008.

6. Voir sur le rapport de ces deux désignations Bernard Dompnier et Paola Vismara (dir.), Confréries et dévotions dans la catholicité moderne ( $m i \mathrm{XV}^{e}$-début XIX $\mathrm{X}^{e}$ siècle), Rome, École française de Rome, coll. «Collection de l'École française de Rome », 393, 2008. Ce recueil offre une excellente synthèse des recherches dans ce domaine, mais dans les limites clairement consenties de l'Europe. Le pas en avant actuellement opéré par Aliocha Maldavsky, dans ses recherches personnelles et au fil de diverses rencontres, consiste à projeter l'histoire des confréries dans les sociétés coloniales, et de montrer quel a été leur rôle pour l'engagement des colons, en particulier des grands proprietaries, à l'avant-poste de l'exploitation des indiens, dans la construction d'une société chrétienne à laquelle ils versaient le rachat de leurs fautes. Elle a aussi établi l'importance décisive des confréries, entre Europe et Amérique, pour le recrutement des missionnaires, souvent liés à des familles impliqués dans l'économie coloniale, à Milan par exemple (voir A.M., "Società urbana e mobilità missionaria: i milanesi e la missione lontana all'inizio del Seicento », in Rivista di storia del Cristianesimo, Brescia, Morcelliana, 2009/1).

7. Signalons également à ce propos les travaux de Jaime Valenzuela sur la présence indienne, d'un repérage difficile par les sources, dans les confréries santiaguines au XVIII ${ }^{\mathrm{e}}$ siècle; voir par exemple «Devociones de inmigrantes. Indígenas andinos y plurietnicidad urbana en la 
conformación de cofradías coloniales (Santiago de Chile, siglo XVII) », Historia, Santiago, Pontificia Universidad Católica de Chile, $n^{\circ}$ 43, vol. I, enero-junio 2010.

8. Voir pour les dernières recherches de Juan Carlos Estenssoro sur les dictionnaires, très tôt constitués dans l'Amérique espagnole, J. Valenzuela éd., XV Jornadas internacionales sobre las misiones jesuiticas, Santiago du Chili, Ediciones de la Universidad Catolica (à paraître en 2015).

9. Silvia Sebastiani, I limiti del progresso. Razza e genere nell'Illuminismo scozzese, Bologne, Il Mulino, 2008 (elle étudie en profondeur la controverse entre Clavijero et Robertson sur la nature de l'écriture dans les codex préhispaniques de «Nouvelle Espagne » et le sens de cette controverse dans la hiérarchisation des civilisations sous l'angle des Lumières européennes).

10. Le stade extrême de cette lecture visuelle étant la conversion esthétique complète de ces manuscrits ou de leurs « retranscriptions », telle qu'elle ressort de la réception de tels documents à Rome par Urbain VIII (voir sur ce point les recherches doctorales en cours, très prometteuses, de Samir Boumediene).

11. On peut rappeler de ce point de vue les études réunies par P. A. Fabre et Bernard Vincent dans Missions religieuses modernes. Notre lieu est le monde, Rome, École française de Rome, 2007, en particulier p. 229-391.

12. Voir désormais sur ce sujet après d'autres travaux le beau livre d'Yves-Marie Bercé, Lorette, Paris, Presses Universitaires de la Sorbonne, 2011.

13. Voir de ce point de vue le brillant et suggestif essai de Jean-Frédéric Schaub, L'Europe a-t-elle une histoire?, Paris, Le Seuil, 2008 - une histoire qui serait d'abord celle de l'autocolonisation d'elle-même. Processus encore inachevé?

14. Je me souviens d'un échange avec Charlotte de Castelnau-Lestoile dans lequel l'«inconstance» qu'elle retenait dans ses Ouvriers d'une vigne stérile comme un trait anthropologique des Indiens du Brésil dans le regard de leurs missionnaires rencontrait celle des spirituels d'Europe, qui en font l'une des marques de leur désenchantement divin, la question se trouvant alors posée de la contamination mutuelle de ces deux diagnostics.

15. Voir de ce point de vue Ines G. Zupanov et P. A. Fabre éds., The Rites Controversy in the Early Modern World (à paraître en 2015).

16. Voir sur le baptême la somme de travaux rassemblée par Adriano Prosperi, Salvezza delle anime, disciplina dei corpi, Pise, Scuola Normale di Pisa, 2006.

17. Patrick Boucheron éd., L'histoire du monde au $X V^{e}$ siècle, Paris, Le Seuil, 2010.

18. Anne Bonzon, «Les curés médiateurs sociaux : genèse et diffusion d'un modèle dans la France du XVII ${ }^{\mathrm{e}}$ siècle ", Revue d'Histoire de l'Église de France, 2011, t. 97, nº 238, p. 35-56.

19. Je me permets de renvoyer sur ce point à l'analyse de ce décret, in P. A. Fabre, Décréter l'image. La XXV Session du Concile de Trente dans le texte, Paris, Belles Lettres, 2013.

20. Cahier des Amériques latines, $\mathrm{n}^{\circ}$ 33, 2000.

21. Les causes américaines restant globalement sinistrées, voir entre autres travaux Antonio Rubial, La santidad controvertida, Mexico, UNAM/FCE, 1999, et récemment René Millar, Santidad, falsa santidad y posesiones demoníacas en Perú y Chile. Siglos XVI y XVII. Estudios sobre mentalidad religiosa, Santiago de Chile, Ediciones Universidad Católica de Chile, 2009.

22. L'œuvre de Jean Séguy est rappelée par Germaine Lemétayer dans sa contribution. La recherche sur les utopies sociales attachées au monde américain peut certainement encore trouver une source d'inspiration chez cet auteur, qui se situait - autre utopie - entre catholicisme et protestantisme, dans ses objets de recherche et sans doute au-delà.

23. Pierre Ragon, Les saints et les images du Mexique (XVI ${ }^{e}$-XVIII ${ }^{e}$ siècle), Paris, L'Harmattan,2003.

24. Jaime Valenzuela l'a bien montré pour les peuples du sud du Chili colonial ; voir par exemple «El uso de la cruz y sus paradojas entre jesuitas y mapuches de la primera mitad del siglo XVII », in Roberto Rusconi y René Millar (eds.), Instituciones, prácticas y representaciones religiosas entre Europa y América Latina (siglos XV-XIX), Rome, Viella, 2010 ; mais aussi dans un tout autre temps et 
contexte Enrique Pilco-Diaz, dans une enquête récente sur les confréries musiciennes de la cathédrale de Cuzco (Des voix dans la pénombre. Le catholicisme cuzquénien à travers les hymnes religieux en quechua. Musique, religion et société dans les Andes du $\mathrm{Xx}^{e}$ siècle, doctorat de l'EHESS, 2010). Ces musiciens-dévots «jouent pour l'image», écrit l'auteur. Que veut dire «jouer pour l'image "? Mais comment comprendre cette "adresse », qui entre en résonance avec un grand nombre de problèmes complexes touchant le dogme et les pratiques cultuelles de l'église catholique dans la longue durée de son histoire? Quelles images encouragent-elles que l'on joue pour elles dans cette cathédrale ? Des images « souffrantes », et qu'il faut soigner par la musique. Ce n'est pas pour n'importe quelle image qu'on joue. Mais la question est d'autant plus sensible que, par ailleurs, la place de l'image dans la parole des hymnes en langue quechua est très limitée, comme l'indique aussi Enrique Pilco. On retire la conclusion que les musiciens sont en quelque manière des médiateurs, non pas seulement des «fidèles » vers les images, mais des images ellesmêmes vers les fidèles, qu'ils les feraient aimer, qu'ils les aideraient à adopter.

25. Serge Gruzinski s'y est récemment essayé avec Quelle heure est-il là-bas ? Amérique et islam à l'orée des Temps modernes, Paris, Le Seuil, 2008, dont la perspective me semble infléchie par rapport à celle, plus surplombante, des Quatre parties du monde : Histoire d'une mondialisation, Paris, Éditions La Martinière, 2004.

26. Il faudrait parvenir à congédier ce terme aujourd'hui envahissant, qui fait d'entrée de jeu du monde un théâtre et piège le discours dans des mirages baroques.

27. Voir pour un développement de cette analyse P. A. Fabre, «Pour une histoire spirituelle des savoirs dans l'espace du monde moderne. Esquisse d'un point de vue », in C. Castelnau-Lestoile, M. L. Copete, A. Maldavsky, I. Zupanov éds., Savoirs missionnaires dans le monde ibérique, Madrid, Casa de Velazquez, 2010.

28. Les travaux récents sur les querelles d'Asie font apparaître la place éminente des rituels funéraires dans l'ensemble du débat, aspect sans doute négligé dans le vaste commentaire de ces querelles à l'époque des Lumières, au profit de la question du nom de Dieu, par exemple. Voir Nicolas Standaert, The interweaving of rituals: funerals in the cultural exchange between China and Europe, Washington, University of Washington Press, 2008.

29. Les points de vues de Foucault sur les ateliers de bienfaisance ont été eux-mêmes multiples, l'accent étant placé tantôt sur l'enfermement (sur un contrôle social), tantôt sur le travail (sur un contrat économique).

30. Voir plus généralement sur les avancées de l'auteur dans ce domaine neuf aujourd'hui d'une histoire culturelle de la théologie, Morales en conflit. Théologie et polémique au Grand Siècle, Paris, Éditions du Cerf, 2011 ; et en co-direction avec Ch.-O. Stiker-Métral, Les Métamorphoses de la théologie. Théologie, littérature et discours religieux au XVII siècle, Paris, Honoré Champion, 2013.

\section{AUTEURS}

\section{PIERRE ANTOINE FABRE}

École des hautes études en sciences sociales, pafabre@ehess.fr 